\title{
Acute Abdominal Pain Revealing a Primary Plasma Cell Leukemia: A Rare and Aggressive Case of Plasma Cell Dyscrasia
}

Luiz Arthur Calheiros Leite ${ }^{1 *}$, Alessandra Gabrielly Magalhães ${ }^{1}$, Jailson Ferreira Silva ${ }^{1}$, Alexssando Pedro Silva ${ }^{2}$, Paloma Lys Medeiros ${ }^{3}$, Jeymesson Raphael Vieira $^{3}$, Cintia Machado ${ }^{4}$, Renata Brandão ${ }^{5}$, Claudia Wanderley Barros Correia ${ }^{5}$, Tómas Jelínek ${ }^{6}$ and Roman Hájek $^{7}$

${ }^{1}$ Department of Hematology, University Hospital Gaffrée and Guinle, Federal State University of Rio de Janeiro, Brazil

${ }^{2}$ Center of Pathology and Diagnostic Medicine, Hospital das Clinicas, Federal University of Pernambuco, Brazil

${ }^{3}$ Department of Histology and Embryology, Federal University of Pernambuco, Brazil

${ }^{4}$ Center of Hematology- HEMOPE, Brazil

${ }^{5}$ Hematology Service, Hospital das Clínicas, Federal University of Pernambuco, Brazil

${ }^{6}$ Department of Haematooncology, University Hospital Ostrava, Ostrava, Czech Republic

${ }^{7}$ Faculty of Medicine, University of Ostrava, Czech Republic

*Corresponding author: Luiz Arthur Calheiros Leite, Department of Hematology, University Hospital Gaffrée and Guinle, Federal State University of Rio de Janeiro, Brazil, Tel: +55 21 2264-4742; Fax: +55 21 2264-5844; E-mail: lahemato@hotmail.com

Received date: Aug 09, 2015, Accepted date: Aug 19, 2015; Published date: Aug 21, 2015

Copyright: ( 2015 Leite LAC, et al. This is an open-access article distributed under the terms of the Creative Commons Attribution License, which permits unrestricted use, distribution, and reproduction in any medium, provided the original author and source are credited.

\begin{abstract}
Primary plasma cell leukemia is a rare and aggressive form of plasma cell neoplasm characterized by the presence of circulating plasma cells in peripheral blood. Here, we report an interesting case of a patient with primary plasma cell leukemia, who was diagnosed during postoperative period after surgery for acute appendicitis. After the appendectomy, the patient developed leukocytosis of $41.2 \times 10^{9} / \mathrm{L}$ with a high number of circulating plasma cells $\left(32.5 \times 10^{9} / \mathrm{L}\right)$. The immunophenotypic analysis of leukemic plasma cells indicated the following antigen profile: $\mathrm{CD} 138^{+++}, \mathrm{CD}^{2} 8^{+++}, \mathrm{IgMc}^{+}, \mathrm{CD}^{-} 6^{-}$and $\mathrm{CD} 20^{-}$. Electrophoresis showed a monoclonal IgG component of 5810 $\mathrm{mg} / \mathrm{dL}$, and diagnosis of primary plasma cell leukemia was established. After the induction chemotherapy with cyclophosphamide, bortezomib and dexamethasone, the patient achieved partial response with regression of hepatomegaly and decreased pleural effusion. Nevertheless, the patient died of sepsis. Therapy with proteasome inhibitors and stem cell transplantation may also provide increased survival and improve the prognosis of patients with primary plasma cell leukemia.
\end{abstract}

Keywords: Plasma cell leukemia; Abdominal pain; Flow cytometry; Bortezomib; Prognosis

\section{Introduction}

Plasma cell leukemia (PPCL) is a rare neoplasm, accounting for less than $5 \%$ of malignant plasma cell diseases [1]. The diagnosis is defined by the presence of more than $2 \times 10^{9} / \mathrm{L}$ circulating plasma cells (PCs) and plasmocytosis $>20 \%$ of total white blood count [2]. The primary form (PPCL) is diagnosed in the leukemic phase without previous diagnosis of multiple myeloma (MM) and accounts for $60 \%$ of all cases of plasma cell leukemia, while secondary PCL (SPCL) arises in the context of a pre-existing refractory or relapsing myeloma disease [3].

Similar symptoms were observed in PPCL and MM patients, such as weakness, fatigue, bone pain, renal impairment, liver enlargement, as well as possible extramedullary involvement (pleural infiltration, cardiac and central nervous system) [4]. Laboratory findings are anemia, thrombocytopenia and increased concentration of serum $\beta 2$ microglobulin $(\beta 2 \mathrm{M})$, high creatinine levels and metabolic abnormalities such as hypercalcemia, elevated lactate dehydrogenase (LDH) levels, and decreased albumin [5].

The circulating clonal PCs are often identified by a strong expression of CD38, CD138 (Syndecan-1), CD20, and weak expression of CD45. Garcia-Sanz et al. [6] showed that antigen CD20 was expressed in $50 \%$ of the cases of PPCL, and this feature is associated with poor prognosis. Additionally, CD56 (neural cell adhesion molecule NCAM) is considered a critical adhesion molecule involved in anchoring of the clonal PCs in bone marrow (BM) [6]. PellatDeceunynck et al. [7] reported that patients with PPCL do not express CD56, and it may be associated with a more aggressive behavior and extramedullary bone marrow dissemination [7]. Studies also showed cytogenetic abnormalities in PPCL patients, such as the deletion of chromosome 13 , and $\mathrm{t}(14 ; 16)[8]$.

The prognosis of PPCL remains poor when compared to MM with a median survival of 8-12 months. The results of conventional chemotherapy are disappointing, but the combination of cyclophosphamide, bortezomib and dexamethasone (CyborD) or bortezomib, dexamethasone and lenalidomid and autologous stem cell transplantation may improve the overall survival of those patients [9].

Here, we report the rare case of a patient with PPCL, who received the diagnosis after surgery for acute appendicitis and hematologic blob smear examination.

\section{Case Report}

Patient MLG, male, 64 years old, born and raised in Pernambuco, sought medical attention in March 2014 with clinical presentation of abdominal fluid accumulation associated with continuous pain on the right and left flank. He denied fever, nausea, vomiting, or other symptoms, had no family history of hypertension, cardiovascular 
disease, stroke or cancer, and he was a former social alcoholic and exsmoker. During the investigation, the diagnosis of acute appendicitis was done and the appendectomy was performed. The biopsy was compatible with acute appendicitis with PCs infiltration. After the surgery, abdominal complaints persisted, referring prevalence of symptoms on the right hypochondrium and associated with asthenia, anorexia, weight loss (9 kg in 2 months), chills and night sweat. During the clinical investigation, the ultrasound of the abdomen and thorax showed a moderate amount of intra-abdominal fluid, bilateral pleural effusion associated with passive atelectasis predominantly on the left side of the chest, and diverticula of the transverse colon and sigmoid. Cardiac evaluation by echocardiography with Doppler also revealed left ventricular segmental variation, aortic stenosis, mitral insufficiency and the electrocardiogram confirmed an atrial fibrillation. Prior to surgery, the blood count showed mild leukocytosis $\left(13.6 \times 10^{9} / \mathrm{L}\right)$, with predominance of neutrophils. However, during the postoperative period, new laboratory tests showed: Complete blood count with increase of leukocytosis $41.2 \times 10^{9} / \mathrm{L}$ (neutrophils, $16 \%$; monocytes, $3 \%$; eosinophils, $3 \%$; and predominance of plasma cells $\left(32.5 \times 10^{9} / \mathrm{L}\right)$, red blood cells $3.15 \times 10^{2} / \mathrm{L}$, hematocrit $28.2 \%$, hemoglobin $9.7 \mathrm{~g} / \mathrm{dL}$, and platelets $93 \times 10^{9} / \mathrm{L}$. The PCs showed basophilic cytoplasm, eccentric nuclei and some binucleated cells (Figure 1). Biochemical tests showed increase of creatinine $(1.8 \mathrm{mg} / \mathrm{dL})$ and urea $(62.7$ $\mathrm{mg} / \mathrm{d} / \mathrm{L}), \gamma$-glutamyltransferase $(685 \mathrm{U} / \mathrm{L})$, alkaline phosphatase $(94$ $\mathrm{U} / \mathrm{L}$ ), lactate dehydrogenase (LDH 521.8 U/L), $\beta 2$-microglobulin (5.57 $\mathrm{g} / \mathrm{mL})$, and decreased albumin $(3.0 \mathrm{mg} / \mathrm{d} / \mathrm{L})$. However, the levels of transaminase, aspartate aminotransferase $(39 \mathrm{U} / \mathrm{L})$, alanine aminotransferase $(29 \mathrm{U} / \mathrm{L})$, and serum calcium were normal (8.5 $\mathrm{mg} / \mathrm{d} / \mathrm{L}$ ). The bone radiography did not show fractures or lytic lesions. Serology for hepatitis B and C, HIV-1 and 2, and syphilis were negative. After that, he was admitted to the Hematology Department for bone marrow (BM) examination, immunophenotyping, and cytogenetic analysis. The BM examination showed hypoplasia of the erythrocyte (3\%) and granulocytic sectors (27\%), megakaryocytic hypoplasia (4\%), and predominance of lymphoplasmacytic cells $(10 \%$ lymphocytes and 56\% plasma cells). Immunophenotyping by flow cytometry revealed a high number of circulating PCs, expressing $\mathrm{CD} 138^{+++}, \mathrm{CD} 38^{+++}, \mathrm{CD}^{+} 5^{+}, \mathrm{IgMc}^{+}$and lambda positive. The other markers CD5, CD19, CD20, FMC7, CD79b, CD117, CD16, CD25, CD10, CD4, CD15, CD56, and kappa were negative (Figure 2). The monoclonal IgG component was $5810 \mathrm{mg} / \mathrm{d} / \mathrm{L} / \mathrm{lambda}$, the Bence Jones protein test was positive and the conventional cytogenetic examination did not show any abnormalities. The diagnosis of primary plasma cell leukemia (PPCL) was confirmed. The patient received four cycles of chemotherapy with CyborD (cyclophosphamide $300 \mathrm{mg} / \mathrm{m}^{2}$, bortezomib $1.5 \mathrm{mg} / \mathrm{m}^{2}$ and dexamethasone, $40 \mathrm{mg}$ on days $1,8,15$ and 22 of a 35 day cycle) resulting in a partial response, followed by reduction of the monoclonal component (IgG, $1200 \mathrm{mg} / \mathrm{dL}$ and disappearance of circulating plasma cells). During the 4 cycles of chemotherapy regimen (D27), the patient presented a night fever at his residence. In the next morning, he was attended by an emergency service that detected dyspnea and hypotension findings (D28), and he was immediately transferred to the intensive care unit of our hospital with pneumonia, septic shock and acute respiratory failure, which started antibiotic therapy with vancomycin $1 \mathrm{~g}$ IV every $12 \mathrm{~h}$ and meropenen $1 \mathrm{~g}$ IV every $8 \mathrm{~h}$ and the CBC showed leukocytes, $0.4 \times$ $10^{9} / \mathrm{L}$, hemoglobin, $10.0 \mathrm{~g} / \mathrm{dL}$ and platelets, $12 \times 10^{9} / \mathrm{L}$. After 24 hours he died to septic shock.

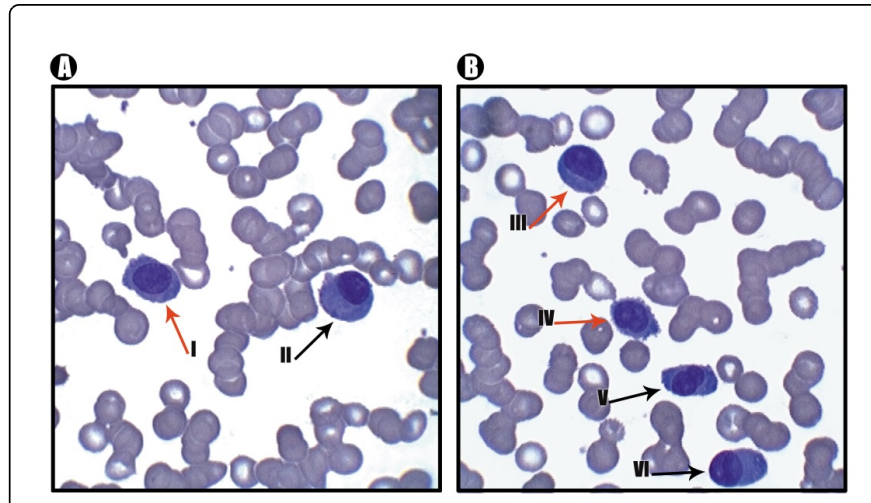

Figure 1: Peripheral blood smear showing marked plasmocytosis with abundant basophilic cytoplasm and eccentrically placed nucleus with clumped chromatin. Binucleate plasma cells also visualized (May-Grünwald-Giemsa staining x 1000).

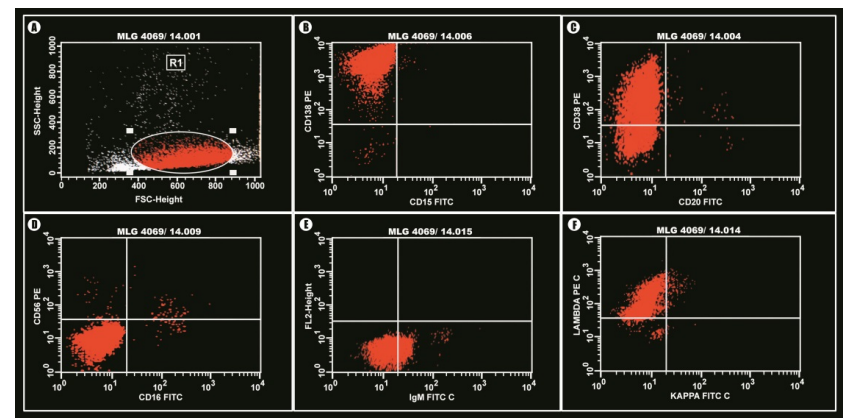

Figure 2: Immunophenotype by flow cytometry of the peripheral blood plasma cells showed the (red) that expressed CD38, CD138, IgMc and cytoplasmic lambda light chain. The antigens CD15, CD16, CD20 and CD56 were negative.

\section{Discussion}

PPCL is defined as a clonal proliferation first diagnosed in the leukemic phase. The current criteria for the diagnosis of PPCL is a clonal proliferation of PCs in the blood comprising more than $20 \%$ of the total leukocytes or an absolute PCs cell count of more than $2 \times$ $10^{9} / \mathrm{L}[1,2]$.

Studies have shown that the main clinical symptoms of patients with PPCL are asthenia associated with severe anemia, thrombocytopenia and extramedular involvement such as hepatomegaly, splenomegaly, lymphadenopathy and other PCs infiltration in the central nervous system, chest with effusion in the pleura and heart, it may be visualized in PPCL patients which can simulate an acute leukemia and diverge from the classic MM [4,5]. Plasmacytomas are more frequent in PPCL with extensive bone disease and overt bone destruction. Hypercalcemia, anemia, thrombocytopenia, increase of LDH, $\beta 2 \mathrm{M}$, creatinine are more frequent at presentation in PPCL when compared with MM $[1,4]$. However, our case did not show lytic lesion or other findings associated with extensive bone disease and the calcium levels were normal, maybe due to the absence of bone disease. 
In our case, the patient also exhibited anemia, thrombocytopenia, increase of $\mathrm{LDH}, \beta 2 \mathrm{M}$, creatinine and during the recovery of surgery, the patient showed an increase of leukocytosis and circulating plasma cells. Moreover, PCs were not detected in the appendices biopsy. This fact demonstrates that the appendicitis may have revealed a PPCL. Pleural effusion and hepatomegaly were also detected and these findings may be associated with infiltration of pleura and liver by the neoplastic plasma cells. Nevertheless, no abnormalities of the liver were detected by ultrasound or computed tomography.

Immunophenotypic analysis of malignant PCs showed strong expression of CD38 and CD138 in all PCL patients. Expression of CD38 is a marker of myeloma cells and it was displayed by leukemic cells in BM, but this antigen is not common in peripheral plasma cells. Few reports confirmed CD38 expression on myeloma cells beyond BM in circulating blood cells or body fluids. On the other hand, the CD56 (NCAM) is responsible for anchoring of PCs on the BM and the absence of this antigen is associated with unfavorable prognosis and aggressive disease. The expression of the lymphocyte antigen CD20 is a common finding of PPCL, however, the CD20 is present in $14 \%$ myeloma cases at diagnosis [6,7]. In our study the immunophenotype by flow cytometry also revealed the presence of high number of circulating plasma cell that expressing CD38, CD138, CD 45, IgMc lambda and CD56 negative. The lack of CD56 (NCAM) in circulating PC may be associated to release of clonal PCs of the BM and should be involved to leukemic transformation. Furthermore, it features a hallmark of leukemic transformation of MM and it is extremely useful for identify the circulating PCs in patients with PPCL.

The immunophenotype by flow cytometry also proved to be extremely indispensable on diagnosis of PPCL, because it was able to characterize the circulating PCs and minimal residual disease. The presence of cytogenetic abnormalities in plasma cell diseases was $30-65 \%$ and our study showed normal cytogenetic analysis. The reason for lack of abnormalities detection on karyotyping could be low proliferation rate of malignant cells, difficulty in obtaining sufficient number of analyzable metaphases, contracted chromosomes resistance of PCs to G-banding and possibly the cells examined cytogenetically were not plasma cells.

In recent years, new drugs such as carfilzomib or bortezomib, lenalidomide, cyclophosphamide and dexamethasone combination have been used for relapsed MM patients and these patients showed a significant improvement with a clinically relevant decrease in the risk of disease progression. Studies suggest that those agents also improve outcome of PPCL, but the benefit may be less pronounced compared with classic MM. Moreover, a retrospective analysis showed that PPCL patients treated with new drugs had a survival of 15 months compared with 8 months for patients who did not receive new agents as part of their treatment $[9,10]$. Bortezomib is probably the most used drug to treat PPCL patients, because bortezomib-based therapy reduces tumor load and reverses complications, including renal damage and hypercalcemia and the combination of bortezomib with other drugs is effective in newly diagnosed PPCL and may be active in refractory PPCL [11]. Drake et al. reports on a very high number of patients with plasma cell leukemia who underwent autologous stem cell transplantation and exhibited a median overall survival of 25.7 months and a progression-free survival of 14.3 months [12].
In our case, the patient initially responded well to chemotherapy with CyborD and achieved a good clinical and laboratory response. Besides, CyborD therapy was effective against malignant PCs infiltration, because after this treatment the patient showed hepatomegaly regression and decrease of pleural effusion. However, the patient died suddenly to septic shock, probably due to immunosuppression associated with chemotherapy.

This report highlights the need of an early recognition of circulating plasma cells to differentiate from other plasma cell dyscrasias and initiate a fast treatment with new therapies such as bortezomib, cyclophosphamide, and dexamethasone in patients with PPCL. Further studies may involve patients with PPCL and MM to understand why patients with PPCL exhibit a more unfavorable prognosis than patients with MM.

\section{References}

1. de Larrea FC, Kyle RA, Durie BG, Ludwig H, Usmani S, et al. (2013) Plasma cell leukemia: consensus statement on diagnostic requirements, response criteria and treatment recommendations by the International Myeloma Working Group. Leukemia 27: 780-791.

2. Kyle RA, Maldonado JE, Bayrd ED (1974) Plasma cell leukemia. Report on 17 cases. Arch Intern Med 133: 813-818.

3. Costello R, Sainty D, Bouabdallah R, Fermand JP, Delmer A, et al. (2001) Primary plasma cell leukaemia: a report of 18 cases. Leuk Res 25 . 103-107.

4. Jimenez-Zepeda VH, Dominguez-Martinez VJ (2009) Plasma cell leukemia: a highly aggressive monoclonal gammopathy with a very poor prognosis. Int J Hematol 89: 259-268.

5. Talamo G, Dolloff NG, Sharma K, Zhu J, Malysz J (2012) Clinical features and outcomes of plasma cell leukemia: a single-institution experience in the era of novel agents. Rare Tumors 4: e39.

6. Garcia-Sanz R, Orfao A, Gonzalez M, Tabernero MD, Bladé J, et al. (1999) Primary plasma cell leukemia: clinical, immunophenotypic, DNA ploidy, and cytogenetic characteristics. Blood 93: 1032-1037.

7. Pellat-Deceunynck C, Barillé S, Jego G, Puthier D, Robillard N, et al. (1998) The absence of CD56 (NCAM) on malignant plasma cells is a hallmark of plasma cell leukemia and of a special subset of multiple myeloma. Leukemia 12: 1977-1982.

8. Avet-Loiseau H, Daviet A, Brigaudeau C, Callet-Bauchu E, Terré C, et al. (2001) Cytogenetic, interphase and multicolor fluorescence in situ hybridization analyses in primary plasma cell leukemia: a study of 40 patients at diagnosis, on behalf of the Intergroupe Francophone du Myeloma and the Groupe Francais de Cytogenetique Hematologique. Blood 97: 822-825.

9. van de Donk NW, Lokhorst HM, Anderson KC, Richardson PG (2012) How I treat plasma cell leukemia. Blood 120: 2376-2389.

10. Stewart AK, Rajkumar SV, Dimopoulos MA, Masszi T, Špička I, et al. (2015) Carfilzomib, lenalidomide, and dexamethasone for relapsed multiple myeloma. N Engl J Med 372: 142-152.

11. Musto P, Rossini F, Gay F, Pitini V, Guglielmelli T, et al. (2007) Efficacy and safety of bortezomib in patients with plasma cell leukemia. Cancer 109: 2285-2290.

12. Drake MB, Iacobelli S, van Biezen A, Morris C, Apperley JF, et al. (2010) Primary plasma cell leukemia and autologous stem cell transplantation. Haematologica 95: 804-809. 